\title{
Supercoiled DNA; plectonemic structure and liquid crystal formation
}

\author{
J R C van der Maarel ${ }^{1}$, S S Zakharova ${ }^{1}$, W Jesse ${ }^{1}$, C Backendorf ${ }^{1}$, \\ S U Egelhaaf ${ }^{2}$ and A Lapp ${ }^{3}$ \\ ${ }^{1}$ Leiden Institute of Chemistry, Leiden University, 2300 RA Leiden, The Netherlands \\ 2 Department of Physics and Astronomy, University of Edinburgh, Edinburgh EH9 3JZ, UK \\ ${ }^{3}$ Laboratoire Léon Brillouin, CEA/CNRS, 91191 Gif-sur-Yvette Cedex, France
}

Received 16 October 2002

Published 16 December 2002

Online at stacks.iop.org/JPhysCM/15/S183

\begin{abstract}
We have investigated the phase behaviour of pUC18 plasmid solutions with phase separation experiments and polarized light microscopy. Furthermore, the configuration of the superhelix is monitored with small-angle neutron scattering. The phase diagram is interpreted with liquid crystal theory including the effects of charge, orientation entropy, excluded volume, as well as the elastic, entropic and electrostatic contributions to the molecular free energy.
\end{abstract}

\section{Introduction}

Closed circular DNA usually exists in a supercoiled configuration, in which the duplex is wound around another part of the same molecule to form a higher-order helix (see figure 1). The plectonemic supercoiling, with a right-handed interwinding, is the most likely conformation in vivo. Supercoiling is utilized in many cellular mechanisms. Examples include the replication and transcription of DNA, the formation of protein complexes on DNA and altered primary structures such as cruciforms.

The organization of DNA in biological structures is largely unknown, but bears some resemblance to liquid crystalline phases observed in vitro [1]. The formation of the anisotropic phase results from the competition between orientation entropy and the volume excluded by a DNA molecule to another molecule [2]. The boundary concentrations are roughly inversely proportional to this excluded volume, which is related to the molecular weight and the range of interaction. In the case of supercoiled DNA, topology is anticipated to be a key factor in controlling the phase behaviour. Apart from its effect on the molecular free energy, the topological constraint sets the dimensions of the superhelix and, hence, determines the excluded volume. Indeed, supercoiling has been reported to be a major compaction mode for plasmid DNA in the cytoplasm of bacteria [3].

Here, we explore the extent to which supercoiling controls the compaction of pUC18 bacterial plasmid (2686 base pairs) in a liquid crystal $[4,5]$. The nature of the phase transition, 


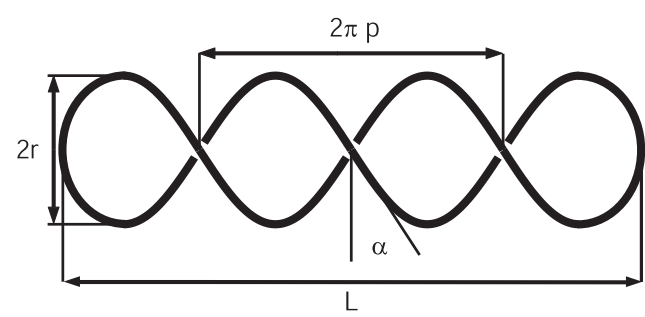

Figure 1. A plectonemic helix with length $L$, radius $r$, pitch $p$ and opening angle $\alpha$.

the critical boundaries and the characterization of the anisotropic phase are investigated with polarized light microscopy and phase equilibrium experiments. Furthermore, the configuration of the superhelix is monitored with small-angle neutron scattering (SANS) through the phase transition. Finally, the phase diagram is interpreted with lyotropic liquid crystal theory including the effects of charge, orientation entropy, excluded volume, as well as the elastic, entropic and electrostatic contributions to the molecular free energy. The effect of the molecular free energy comes in through the effect on the balance of chemical potentials pertaining to the coexisting phases.

\section{Topology}

The topological constraint is characterized by the linking number deficit $\Delta L k$, which is the number of turns the duplex is turned by before closure to form a ring. As shown by gel electrophoresis, our DNA preparation contains a broad topoisomer distribution with predominant $\Delta L k=-7$ and deviation \pm 3 [4]. $\Delta L k$ is conserved and is distributed between the writhe $W r$ and excess twist $\Delta T w$ exerted on the duplex according to [6]

$$
\Delta L k=W r+\Delta T w .
$$

For a right-handed, regular supercoil without end loops, $W r$ is proportional to the number of crossings $n$ when viewed perpendicular to the superhelical axis: $W r=-n \sin \alpha$, with the pitch angle $\alpha$ as in figure 1 . It is convenient to define the normalized length $2 L / l$, with $l$ being the length of the DNA molecule. From integration along the contour it follows that

$$
2 L / l=p /\left(p^{2}+r^{2}\right)^{1 / 2},
$$

if the end loops are neglected. The pitch angle $\alpha$ is given by

$$
\tan \alpha=p / r=(2 L / l) /\left(1-(2 L / l)^{2}\right)^{1 / 2}
$$

and the writhe reads

$$
W r=-l p /\left(2 \pi\left(p^{2}+r^{2}\right)\right) .
$$

The local structure of the superhelix is fully characterized by $p$ and $r$. These parameters determine $\alpha, W r$ and $2 L / l$. At a larger length scale, the superhelix can branch with an average of 1.2 branch points per molecule [7].

\section{Phase diagram}

If the plasmid concentration is increased beyond a certain critical concentration, the translucent solution is seen to be composed of birefringent domains when observed through crossed polarizers. Polarized light microscopy textures show that this observation is due to the 

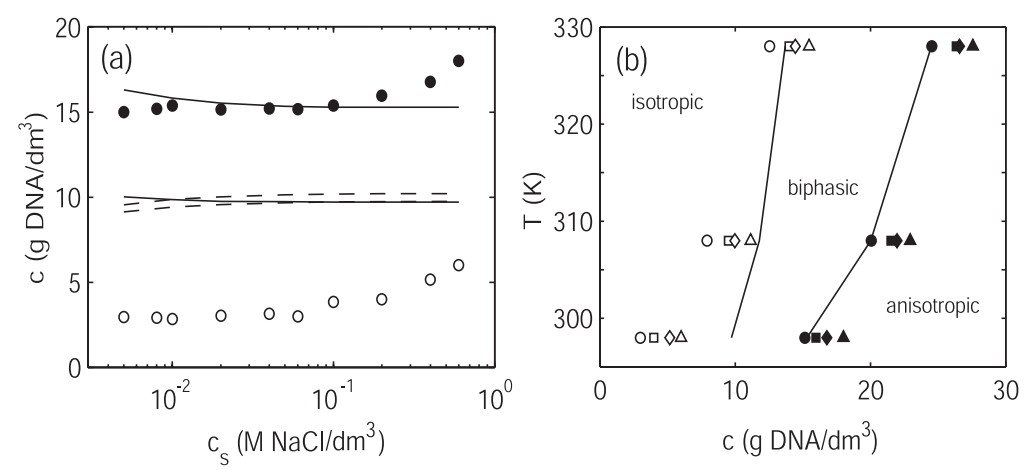

Figure 2. (a) Critical boundaries versus salt concentration $(T=298 \mathrm{~K})$. Open symbols: $c_{i}$; closed symbols: $c_{a}$. The curves are calculated with the scaled particle description for hard-core effects and electrostatic interactions in the second virial approximation without (dashed curves) and with (solid curves) a change in molecular configuration through the phase transition. (b) The temperature-dependent phase diagram for $0.1(\square), 0.2(\triangle), 0.4(\diamond)$ and $0.6 \mathrm{M}(\triangle) \mathrm{NaCl}$.

formation of a liquid crystal rather than aggregation phenomena. In addition to light microscopy, macroscopic phase separation experiments were carried out to obtain a precise estimate of the phase boundaries.

For phase equilibrium experiments, biphasic samples with coexisting isotropic and liquid crystalline phases were prepared. The volume fraction of the anisotropic phase increases linearly from zero for isotropic samples to unity for the liquid crystal. The critical boundaries were obtained by extrapolation to the concentrations at which the anisotropic phase first appears $c_{i}$ and the isotropic phase completely disappears $c_{a}$, respectively. As shown in figure 2, the transition is strongly first order with a broad gap between the phase boundaries. For concentrations below, say, $0.1 \mathrm{M} \mathrm{NaCl}$, the boundaries are rather salt concentration independent; for higher salt concentrations they show a small increase with increasing ionic strength. This behaviour is very different from the one observed for linear DNA, where the phase diagram shows a relatively narrow phase gap and a strong dependence on ionic strength. Furthermore, for the supercoil the boundaries are significantly lower than for linear DNA [8].

In contrast to the weak salt dependence, the boundaries are very sensitive to temperature. At a sufficiently high, fixed DNA concentration, it is possible to convert the anisotropic into the isotropic phase by raising the temperature; the liquid crystal reappears once the solution is cooled below the clearing point. We have checked that this clearing effect is fully reversible, so there are no irreversible changes involved. The clearing point depends on overall DNA concentration as shown in figure 2. The temperature dependence of the phase diagram is very different from the one observed for $50 \mathrm{~nm}$ length linear DNA, where the boundaries were observed to be weakly dependent on temperature only [9].

The assignment of the anisotropic phase has caused some confusion in the literature, because non-aligned samples do not show the characteristic fingerprint-like textures expected for a cholesteric molecular organization (see figure 3(a)) [3, 10]. Due to the negative diamagnetic susceptibility of the bases, DNA arranges with its long axis perpendicular to the direction of a magnetic field [11]. For linear DNA, this effect can be used to induce macroscopic alignment [12]. However, supercoiled DNA has a very weak diamagnetic anisotropy, because the axis of the duplex follows a classical plectonemic trajectory such that the anisotropy of the bases averages out [10]. We have succeeded in inducing macroscopic alignment by exposing the preparation slide for more than $12 \mathrm{~h}$ in a $14 \mathrm{~T}$ field. As displayed in figure 3(b), a typical 
(a)

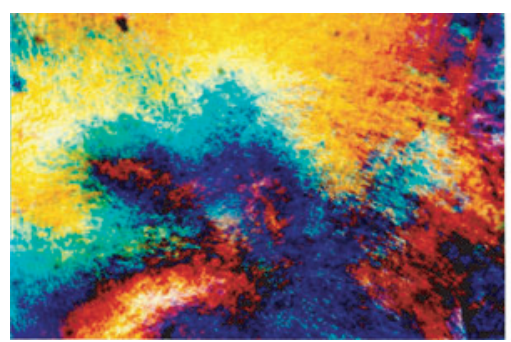

(b)

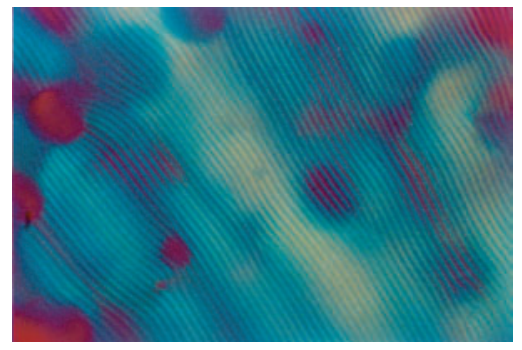

Figure 3. Polarized light microscopy textures for $15 \mathrm{~g}$ of $\mathrm{pUC} 18 \mathrm{dm}^{-3}$ in $0.1 \mathrm{M} \mathrm{NaCl}$. (a) Nonaligned; $1000 \times$. (b) As (a), but after prolonged exposure to a $14 \mathrm{~T}$ magnetic field with direction parallel to the slide with the result that the cholesteric axis is perpendicular to the line of view.

(This figure is in colour only in the electronic version)
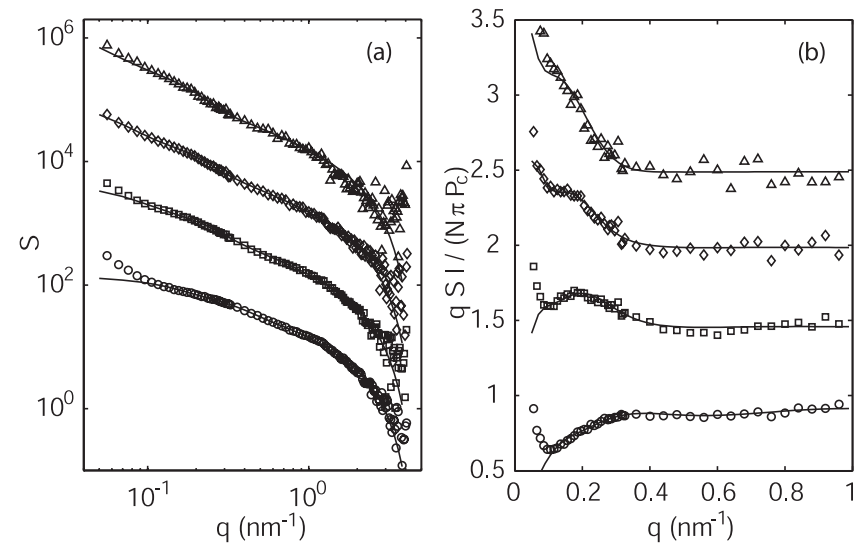

Figure 4. (a) Structure factor $S$ versus momentum transfer $q$ for $3(\triangle), 6(\diamond), 11(\square)$ and $27 \mathrm{~g} \mathrm{DNA}^{-3}$ (O) in $0.05 \mathrm{M} \mathrm{NaCl}$. (b) As (a), but for the normalized structure factor. Curves represent a fit with parameters in table 1 . To avoid overlap, the data are shifted along the $y$-axis.

cholesteric, fingerprint-like texture is now observed, provided that the direction of the magnetic field was parallel to the slide with the result that the cholesteric axis is oriented perpendicular to the line of view. The cholesteric pitch, measured for twice the distance between the fringes, amounts to $4 \mu \mathrm{m}$, which is about twice the value observed for linear DNA [9].

\section{Neutron scattering}

SANS from pUC18 plasmid in $0.05 \mathrm{M} \mathrm{NaCl}$ was measured at the Institute Laue-Langevin, Grenoble, and Laboratoire Léon Brillouin, CE de Saclay, France, and the results are displayed in figure 4.

In our experiments, the range of momentum transfer $q$, which is defined by the wavelength $\lambda$ of the radiation and the angle $\theta$ between the incident and scattered beam according to $q=4 \pi / \lambda \sin (\theta / 2)$, exceeds $L^{-1}$ by at least an order of magnitude. In this high- $q$ range, the scattering is sensitive to interference over an extent of the order of $r$ and $p(q r \approx 1$ and $q p \approx 1)$ and effects of overall flexibility and/or branching of the supercoil are beyond observation. We 
Table 1. Parameters resulting from the fit of the structure factor to the scattering data. Note that the margins are related to a variation in molecular shape.

\begin{tabular}{lrrllll}
\hline$c\left(\mathrm{~g} \mathrm{dm}^{-3}\right)$ & $r(\mathrm{~nm})$ & $p(\mathrm{~nm})$ & $\alpha(\mathrm{deg})$ & $2 L / l$ & $W r$ & $A_{2}\left(10^{6} \mathrm{~nm}^{3}\right)$ \\
\hline 3 & $10 \pm 4$ & $21 \pm 9$ & 65 & 0.91 & $-6 \pm 3$ & - \\
6 & $9 \pm 5$ & $14 \pm 7$ & 57 & 0.84 & $-7 \pm 4$ & 0.55 \\
11 & $8 \pm 4$ & $13 \pm 7$ & 59 & 0.86 & $-9 \pm 5$ & 1.22 \\
27 & $5 \pm 3$ & $6 \pm 4$ & 52 & 0.79 & $-14 \pm 10$ & 1.92 \\
\hline
\end{tabular}

can accordingly use the high- $q$ approximate form of the form factor of a regular superhelix [4]:

$$
P(q)=\frac{\pi}{q L}\left[J_{0}^{2}(q r)+2 \sum_{k=1}^{q p / 2} J_{2 k}^{2}\left(\left(q^{2}-4 k^{2} / p^{2}\right)^{1 / 2} r\right)\right], \quad q L \gg 1 .
$$

The effect of the cross-sectional radius of gyration $r_{p}$ of the duplex is taken into account by multiplication of equation (5) with the form factor pertaining to the cross-section:

$$
P_{c}(q)=\left[2 J_{1}\left(q r_{p}\right) /\left(q r_{p}\right)\right]^{2} .
$$

Note that $P(q)$ is sensitive to the DNA density per unit length projected on the superhelical axis $L^{-1}$. However, for $q r \gg 1$ and $q p \gg 1$ the scattering is essentially given by a single strand of the superhelix, which is proportional to the density per unit contour length $l^{-1}$. In both regimes, the form factor displays the characteristic $q^{-1}$-scaling for rodlike particles, but the prefactor drops from $L^{-1}$ to $l^{-1}$. As we will see shortly, our data do not comply with such idealized structure as depicted in figure 1. We will accordingly assume a Gaussian distribution in $r$ with standard deviation $\sigma_{r}$ and a distance of closest approach of the two opposing strands in the superhelix $2 r_{m}\left(r_{m} \approx 2 \mathrm{~nm}\right)$. It is also assumed that $\alpha$ is constant, which implies that $p$ is proportional to $r$ with a proportionality factor given by equation (3). We have checked that a model in which $p$ and $r$ are allowed to fluctuate independently does not give a better fit. Interactions among supercoils are accounted for in the second virial approximation and the total scattering function takes hence the form

$$
S(q)=N P(q) /\left(1+2 A_{2} \rho P(q)\right),
$$

with $N$ the number of bases, $A_{2}$ the second virial coefficient and $\rho$ the DNA density.

The effect of interactions among supercoils on the structure factor is vanishing small for $\rho$ less than, say, $3 \mathrm{~g}$ of DNA dm$~_{-3}$ (i.e. $\rho A_{2} \approx 0$ ). For samples with higher plasmid concentration, progressive interactions result in a suppression of the intensity at smaller $q$ values. The agreement with second virial theory and the absence of an interaction peak indicate that the supercoils do not interact too strongly. Due to the presence of a significant distribution in $r$ and $p$, the structure factors do not exhibit strong oscillatory behaviour. They do show, however, the anticipated $q^{-1}$-scaling and the drop in prefactor from $L^{-1}$ to $l^{-1}$ with increasing $q$. For $q$ exceeding, say, $1 \mathrm{~nm}^{-1}$, the structure factors show the characteristic deviation from $q^{-1}$-scaling related to the cross-section of the duplex with $r_{p}=0.8 \mathrm{~nm}$.

The plectonemic structure is most clearly demonstrated in figure 4(b), where the structure factors are normalized in such a way that they go to unity at high $q$. The normalized structure factor extrapolates to $2 l / L$ for $q \rightarrow 0$ and in the absence of interactions. In the fit procedure, we have optimized $r$, its distribution width $\sigma_{r}, \alpha$ and $A_{2}$. The other parameters are derived through equation (3) and standard variance propagation. Note that the margins are not related to error, but rather to variation in molecular shape. The main contribution comes from the presence of a distribution of topoisomers. Other sources are thermal fluctuations and partitioning of DNA over coexisting isotropic and liquid crystalline phases. 
With increasing concentration through the phase transition, $r$ and $p$ are seen to decrease significantly. As a result of the (near) constancy of $\alpha, W r$ decreases and the number of superhelical turns increases $(W r=-n \sin \alpha$ ). According to the fact that through equation (1) the sum of the excess twist and the writhe are conserved, this decrease in $W r$ should be compensated by a positive twist exerted on the duplex if $\Delta L k$ is conserved. Apart from the change in physical size of the supercoil, the associated increase in molecular free energy is of great importance in controlling the phase boundaries.

\section{Discussion and conclusions}

The phase behaviour is explained by considering the interplay between excluded volume and orientation order of a system of uniformly charged, wormlike cylinders. To decrease excluded volume, the system increases anisotropy with a concurrent decrease in size of the supercoil. With a bending persistence length of the supercoil around $100 \mathrm{~nm}$, we are in the very flexible chain limit and effects of overall flexibility are expected to be moderate. Due to counterion condensation, the effective charge of the supercoil is quite low. The electrostatic contribution is accordingly evaluated as a thermodynamic perturbation in the second virial approximation, whereas the hard-core contribution is evaluated with scaled particle theory to include higherorder terms [13]. In the analysis we will neglect branching of the supercoil, as its effect on the phase boundaries is unknown.

We first assumed that the topology does not change through the phase transition, i.e. the boundaries are calculated with $r=10 \mathrm{~nm}$ and $\alpha=65^{\circ}$ pertaining to the isotropic phase. As shown by the dashed curves in figure 2 , the conventional narrow phase gap is obtained. The predictions can be improved if in the liquid crystal $r$ and $\alpha$ are reduced to $7.5 \mathrm{~nm}$ and $58^{\circ}$, respectively (solid curves), in accordance with the scattering results. Due to the decrease in excluded volume and increase in molecular free energy through the effect on the chemical potentials, $c_{a}$ is shifted towards higher concentrations and is now in reasonable agreement with the data. The prediction for $c_{i}$ is unaffected, however, because the dimensions in the isotropic phase were kept at their original values. With increasing temperature, the boundaries shift to higher concentrations, which indicates that the excluded volume decreases. Indeed, the shift can be reproduced by a small decrease in $r$. The solid curves in figure 2(b) connect the predictions with optimized $r=7.5,6.9$ and $6.4 \mathrm{~nm}$ at 298, 308 and $328 \mathrm{~K}$, respectively, so $c_{a}$ is reproduced.

The broadening of the phase gap can be partially explained by theory including a decrease in size of the supercoil through the phase transition. The theoretical results compare favourably with $c_{a}$, but they fail to predict $c_{i}$. This is probably related to branching in the isotropic phase, which results in an increase in excluded volume and shift of the corresponding boundaries towards lower concentrations. In the liquid crystal, branching is presumably suppressed due to the spatial confinement. An explanation for the thermal phase diagram can be found in the twist energy storing property of the supercoil and changes in flexibility of the duplex. With increasing temperature, the flexibility increases due to increased breathing of the base pairs. As a consequence of the associated decrease in elastic energy, the superhelix can be more tightly interwound, which results in a smaller excluded volume and a reversible shift of the boundaries towards higher packing fractions.

\section{References}

[1] Livolant F and Leforestier A 1996 Prog. Polym. Sci. 211115

[2] Onsager L 1949 Ann. NY Acad. Sci. 51627 
[3] Reich Z E, Wachtel J and Minsky A 1994 Science 2641460

[4] Zakharova S S, Jesse W, Backendorf C, Egelhaaf S U, Lapp A and van der Maarel J R C 2002 Biophys. J. 83 1106

[5] Zakharova S S, Jesse W, Backendorf C and van der Maarel J R C 2002 Biophys. J. 831119

[6] White J H 1969 Am. J. Math. 91693

[7] Boles T C, White J H and Cozzarelli N R 1990 J. Mol. Biol. 213931

[8] Kassapidou K, Jesse W, van Dijk J A P P and van der Maarel J R C 1998 Biopolymers 4631

[9] Rill R L, Strzelecka T E, Davidson M W and van Winkle D H 1991 Physica A 17687

[10] Torbet J and DiCapua E 1989 EMBO J. 84351

[11] Maret G, Schickfus M V, Mayer A and Dransfeld K 1975 Phys. Rev. Lett. 35397

[12] Brandes R and Kearns D R 1986 Biochemistry 255890

[13] Sato T and Teramoto A 1996 Adv. Polym. Sci. 12685 